\title{
Accuracy of ultrasonography in detecting gastrointestinal tract obstructions in children; a prospective study in a tertiary referral hospital
}

\author{
Parisa Hajalioghli ${ }^{1}$, Saeid Aslanabadi ${ }^{2}$, Mostafa Vafaei Aghdam¹, Daniel Fadaei Fouladi ${ }^{3}$
}

${ }^{1}$ Department of Radiology, Imam Reza Teaching Hospital, ${ }^{2}$ Department of Pediatric Surgery, Koodakan Teaching Hospital, ${ }^{3}$ Neurosciences Research Center, Tabriz University of Medical Sciences, Tabriz, Iran

\begin{abstract}
Aims: To examine the diagnostic accuracy of plain radiography, abdominal ultrasonography (US), and their combination in pediatric patients with suspected gastrointestinal (GI) tract obstruction. Material and methods: A cohort of 48 patients (age, 0-14 years, 27 boys) with clinical manifestations of GI tract obstruction underwent plain radiography and abdominal US examination. The final diagnoses were based on intraoperative findings, rectal biopsies (in Hirschsprung's disease), or adequate follow-ups. Results: The GI tract obstruction was diagnosed in 40 patients. The sensitivity, specificity, positive predictive value and negative predictive value of plain radiography in diagnosing GI tract obstruction were $87.5 \%, 75.0 \%, 94.6 \%$, and $54.6 \%$, respectively. The corresponding values were $95 \%, 100 \%, 100 \%$, and $80 \%$, respectively when US was used alone; and $97.5 \%, 100 \%, 100 \%$ and $88.9 \%$, respectively when radiography and US were used together. Except for two patients (one with Hirschsprung's disease and the other with massive peritonitis), US detected the underlying causes of obstruction correctly in all patients. Conclusions: US is a highly sensitive and specific modality in diagnosing pediatric GI tract obstructions, as well as their causes. The combination of plain radiography and US further increase the diagnostic sensitivity and negative predictive value.
\end{abstract}

Keywords: ultrasonography; radiography; intestinal obstruction; pediatrics

\section{Introduction}

Gastrointestinal tract obstructions are relatively common in children. Stenosis, atresia, annular pancreas, adhesion bands and webs, malrotation and volvulus, ileus and Hirschsprung's disease are among the usual causes of pediatric GI tract obstructions [1,2].

In general, obtaining the history and abdominal radiography constitute the mainstay of diagnosis in children with suspected GI tract obstruction. This strategy,

Received 17.08.2019 Accepted 19.12.2019

Med Ultrason

2020, Vol. 22, No 2, 139-144

Corresponding author: Daniel F. Fouladi, MD

Neurosciences Research Center, Tabriz

University of Medical Sciences, Tabriz, Iran

Phone: +98 4133340730

E-mail: dfoulad1@jhmi.edu however, is not always accurate or adequate. So, better diagnostic approaches, which are fast, noninvasive and precise are helpful. Computed tomography (CT) and magnetic resonance imaging (MRI) provide the most accurate information when GI tract obstructions are suspected. Limited access, cost, radiation hazard (for CT) and the need for child sedation during imaging are the main drawbacks of these modalities. So, they are not considered routinely as the initial imaging studies when the GI tract obstruction is suspected [3-5].

Some authors have suggested abdominal ultrasonography (US) as a noninvasive, simple and widely available imaging technique in examining patients with suspected GI tract obstruction [6-10]. Small body habitus and the absence of abundant fat tissue in the peritoneal cavity and abdominal wall suggest the US as an accurate modality in assessing intraabdominal pathologies including GI tract obstruction in neonates and pediatric patients 


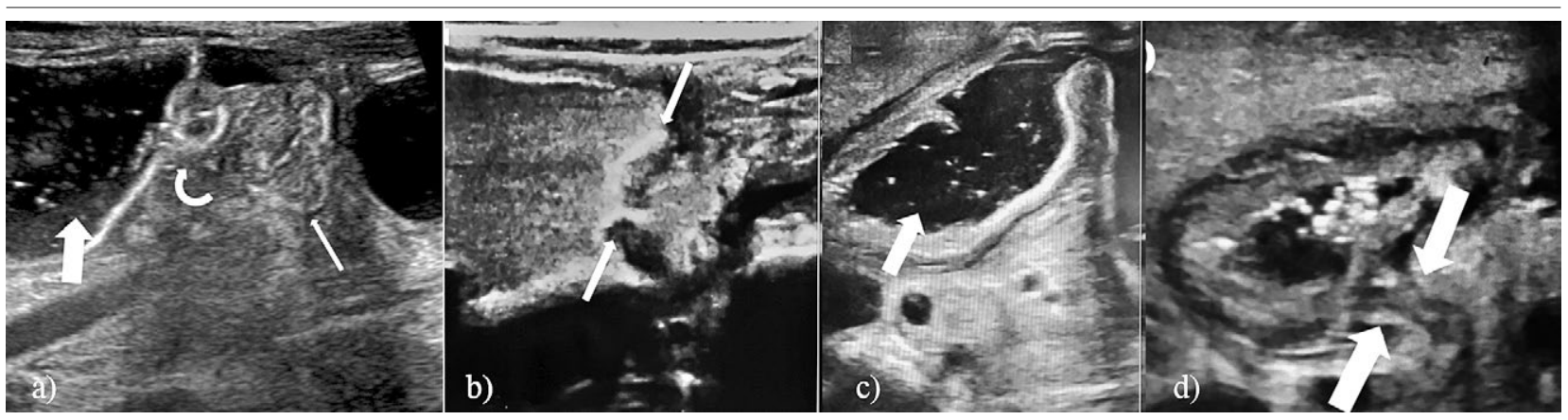

Fig 1. A 2.5-year-old girl with dilated (thick arrow) and decompressed (thin arrow) intestinal loops. Note the transition point (curved arrow) developed by an adhesion band due to a previous surgery in that area (a). A 3-year-old girl with the dilated third part of the duodenum and a thick web in the transition point (arrows) (b). A 6-year-old boy with obstruction in the third part of the duodenum (arrow) (c). The transition point (arrows) is developed by an annular pancreas (d).

$[11,12]$. Related reports in children, however, are scarce and therefore, the true accuracy of the US is yet to be defined.

The objective of this study was to determine the diagnostic performance of the abdominal US in detecting pediatric GI tract obstructions and their underlying causes in a tertiary referral hospital.

\section{Material and methods}

\section{Study design and patients}

A total of 48 children (age, $0-14$ years) with clinical manifestations of GI tract obstruction were enrolled in this prospective study in a university-affiliated, tertiary referral children hospital within two years. The inability to perform US exam and hemodynamical instability were the exclusion criteria.

This study was approved by the Ethics Committee of our university. Informed consent was obtained from parents or legal guardians, according to the World Medical Association Declaration of Helsinki, revised in 2000, Edinburgh.

\section{Imaging studies}

All patients underwent the routine protocol of the management of patients with suspected GI tract obstruction in our hospital. An attending radiologist with 10 years of academic experience in pediatric sonography performed abdominal US examinations using a standard machine (Ultrasonix OP, Ultrasonix Medical Corporation, British Columbia, Canada) equipped with linear 5-14 MHz transducers. With the patient placed in the supine position, the transducer was moved transversely over the abdominal quadrants. In crying patients, the exams were completed between crying spells or when the child calmed down in the parent's arms. A GI tract obstruction was suspected when a transition point (an abrupt transition from proximal dilated loops to distal decompressed loops) [11] was visualized during the US examination (fig1).

Hirschsprung's disease was reported when the rectosigmoid index was less than 1 [13] (fig 2a) and intestinal malrotation when the whirlpool sign (swirling appearance of the superior mesenteric vein around the superior mesenteric artery) was present [14] (fig 2b).

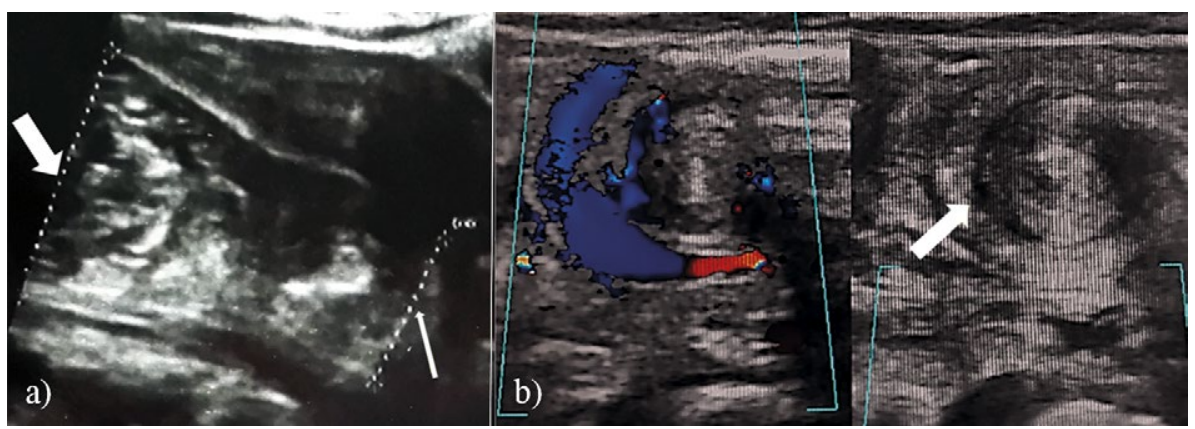

Fig 2. a) The rectosigmoid index was measured as the diameter of the rectum (thin arrow) divided by the diameter of the sigmoid colon (thick arrow) in this 1.5-year-old girl. The rectosigmoid index was $<1$ in this case, suggesting the diagnosis of Hirschsprung's disease; b) A 2-year-old girl with malrotation and positive whirlpool sign. Note the swirling appearance of the superior mesenteric vein (arrow) around the superior mesenteric artery. 
To differentiate meconium ileus from ileal atresia the echogenicity of the intestinal loop was noted. In meconium ileus, the intestinal loops usually contain dense echogenic materials, whereas in atresia the fluid within the loops is often hypoechogenic [15]. To separate mechani$\mathrm{cal}$ and functional obstructions, changes in the intestinal motility were monitored by time. Generally, the intestinal movements increase initially when a mechanical obstruction is present, but finally, the involved segment becomes ischemic and immotile. Loop akinesia was reported when peristalsis was absent after 5 minutes of continuous observation [16].

On the same day, all patients underwent upright chest and supine/standing plain abdominal radiography. Fluoroscopic studies had been performed at the discretion of the referring physician in 15 patients. Another radiologist with 4 years of academic experience and blind to the results of the US examination reviewed the radiographs. Finally, the two radiologists reached a consensus regarding the presence and the etiology of GI tract obstruction after reviewing radiographs and US results side by side.

\section{Standard of criterion}

The final diagnosis was based on intraoperative findings and/or the results of rectal biopsy in the 40 patients. The remaining 8 patients were monitored in the hospital and followed up after discharge.

\section{Statistical analysis}

The SPSS software for Windows V 16.0 (SPSS Inc., IL, USA) and Microsoft Excel were used for statistical analysis and calculation of the sensitivity, specificity, positive predictive value (PPV), and negative predictive value (NPV), respectively.

\section{Results}

Patients' characteristics and general information are summarized in Table I.

GI tract obstructions were confirmed in 40 patients: 11 with Hirschsprung's disease, 6 with ruptured appen-

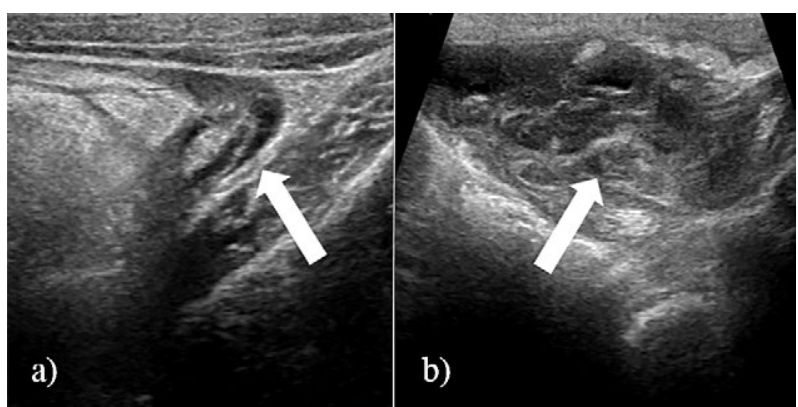

Fig 3. A 7-year-old boy with ruptured appendicitis (arrow) (a) and the surrounding collection (arrow) (b)
Table I. Patients' characteristics and general data

\begin{tabular}{lll}
\hline Datum & & No $(\%)$ \\
\hline Sex & Male & $27(56.3)$ \\
Age & Female & $21(43.8)$ \\
& $\leq 1$ month & $29(60.4)$ \\
& $>1$ month, $<1$ year & $9(18.8)$ \\
& $\geq 1$ year, $<5$ years & $6(12.5)$ \\
Presenting symptom & $\geq 5$ years & $4(8.3)$ \\
& Vomiting & $14(29.2)$ \\
& Obstipation & $12(25)$ \\
& Abdominal distension & $8(16.7)$ \\
& Abdominal pain & $8(16.7)$ \\
& Constipation & $3(6.3)$ \\
& Hematemesis & $3(6.3)$ \\
\hline
\end{tabular}

dicitis, 6 with ruptured appendicitis (fig 3), 4 with annular pancreas, 4 with duodenal adhesion, 4 with intestinal malrotation, 3 with duodenal atresia, 3 with duodenal web, 2 with intestinal adhesion, 2 with intestinal atresia (fig 4) and 1 with internal abdominal hernia.

GI tract obstructions were ruled out in the remaining 8 patients after conservative management and adequate follow-ups.

Fluoroscopic studies were performed in 15 patients. Accordingly, GI tract obstruction was ruled out correctly in 2 patients and diagnosed correctly in 12 patients, including 7 patients with Hirschsprung's disease and 5 patients with small bowel obstruction. In 1 patient the result was "undetermined diagnosis".

\section{Plain radiography}

By using radiography alone, GI tract obstruction was diagnosed correctly in 35 patients $(72.9 \%)$ patients and incorrectly in 2 patients (4.2\%) patients. GI tract obstruction was ruled out correctly in 6 patients $(12.5 \%)$ and missed in 5 patients (10.4\%).

\section{Ultrasonography}

By US examination alone, GI tract obstruction was diagnosed correctly in 38 patients $(79.2 \%)$, ruled out correctly in 8 patients $(16.6 \%)$ and missed in 2 patients (4.2\%). There was no false-positive finding when US was used. The underlying causes of GI tract obstruction were detected correctly in 38 out of 40 patients (95\%) with GI tract obstructions. There was one patient with Hirschsprung's disease (15-month-old girl), who was wrongly reported with ileus. The obstruction was missed during the US examination in another patient (11-yearold girl) with ruptured appendicitis and concomitant peritonitis.

\section{Plain radiography plus ultrasonography}

The GI tract obstruction was detected in the patient with peritonitis when radiographs and US findings were 


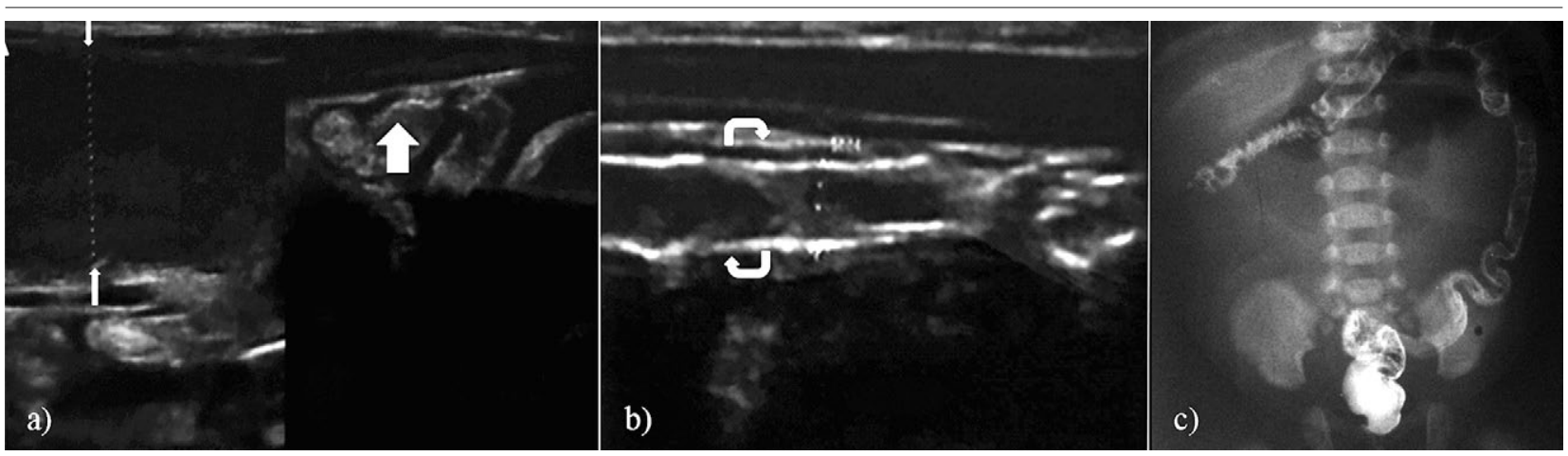

Fig 4. A 35-day-old boy with ileal atresia. Note the dilated proximal small bowel loop (thin arrows), decompressed distal ileal loop (thick arrow) (a) and the narrowed colon distal to the atresia (curved arrows) (b). Barium enema shows microcolon in this patient (c).

examined side by side. So, GI tract obstruction was diagnosed correctly in 39 patients $(81.3 \%)$, ruled out correctly in 8 patients $(16.6 \%)$ and missed in 1 patient $(2.1 \%)$. There were no false-positive findings when radiography images and US results were combined.

In table II the diagnostic performances of the plain radiography, US and combined methods in detecting gastrointestinal tract obstruction in children are summarized.

\section{Discussion}

In this study, we showed that US is a highly sensitive $(95 \%)$ and specific $(100 \%)$ imaging modality in both diagnosing GI tract obstructions and detecting the underlying causes of the GI tract obstructions in children. A combination of plain radiography and US further increased the diagnostic accuracy, especially in patients with conditions such as peritonitis, in which US alone may fall short.

Delay in the diagnosis of GI tract obstructions in children could dramatically increase morbidity and mortality rates [17]. In a classic approach, patients with suspected GI tract obstructions are managed on the basis of clinical and radiographic findings [18]. In children, however, clinical manifestations are sometimes vague and unreliable [19] and radiographic findings, as seen in the present study, are often nonspecific [20]. Previous studies have reported US as an accurate modality in the diagnosis of
GI tract obstructions in adults [5,16,18,21]. In detecting bowel obstructions, for instance, US has been found to be $95 \%$ sensitive and $>80 \%$ accurate $[22,23]$.

Causes of GI tract obstructions, however, are different in the adult population. GI tract obstructions in children represent a very heterogeneous group of conditions, involving all segments of the GI tract and including congenital malformations and anomalies. The diagnostic approach depends on the level of the obstruction, its mechanism or the age of the patient.

Studies in children are rare and limited to particular sites in the GI tract $[15,24,25]$. For example, Ikeda et al [24] found US useful in identifying underlying causes of small bowel obstructions in neonates younger than 1 month old. In a small case series, James et al [25] used point-of-care US successfully in the diagnosis of small bowel obstruction in five symptomatic children. In contrast, Maheshwari et al [15] found US inaccurate in detecting underlying causes of obstructions in the distal parts of GI tract in children.

Compared to adults, the body habitus of children are smaller and there is less fat in their peritoneal cavity and abdominal wall. These advantages can increase the accuracy of US in assessing intraabdominal pathologies in neonates and pediatric patients [12]. Detecting obstructions in the small bowel is easy because fluid within the dilated loops proximal to the transition point serves as a contrast medium during the US examination. Moreover,

Table II. Diagnostic performance of plain radiography, ultrasonography (US) and combined plain radiography and US in detecting gastrointestinal tract obstruction in children

\begin{tabular}{llll}
\hline Variable (\%) & Radiography & US & Radiography plus US \\
\hline Sensitivity & $87.5(73.2-95.8)$ & $95.0(83.1-99.4)$ & $97.5(86.8-99.9)$ \\
Specificity & $75.0(34.9-96.8)$ & 100.0 & 100.0 \\
Positive predictive value & $94.6(81.8-99.3)$ & 100.0 & 100.0 \\
Negative predictive value & $54.6(23.4-83.3)$ & $80.0(44.4-97.5)$ & $88.9(51.8-99.7)$ \\
\hline
\end{tabular}

Figures in parenthesis represent $95 \%$ confidence interval. 
the fluid-filled bowel may act as a sonographic window that facilitates the diagnosis of the underlying causes of the obstruction [11].

Ruptured appendicitis was a rather frequent cause of GI tract obstruction in the present study. Because of their inability to communicate their symptoms, the perforation rates of appendicitis are high among neonates $(83 \%)$ and young children (51-100\%) [26]. The increased diameter of the appendix, incompressibility and adipose hyperechogenicity sign are three main criteria in diagnosing appendicitis using abdominal US [27].

US is an operator-dependent technique and the experience of the sonographer plays an important role in determining the accuracy of US when a specific condition is being assessed [28].

We had only two missed cases when US was used; one patient with ruptured appendicitis and extensive peritonitis that prevented adequate visualization of the abdominal cavity and the second patient with Hirschsprung's disease. Although US could be still useful in examining patients with peritonitis $[29,30]$, due to the presence of pneumoperitoneum and gaseous artifacts, it may fail to identify the cause of bowel obstruction [30,31]. In such cases, US combined with radiographs could be helpful [32], as in our study.

This study bears three limitations. First, the majority of patients were under 5 years of age, making extrapolation of results to all age groups of children difficult. Further studies with larger sample sizes including older children are recommended. Second, only one sonographer was present in our study and this prevented us from calculating interobserver agreement. Third, this study was performed in a tertiary referral hospital and so many patients suffered surgically treatable causes of obstruction. To get more accurate results particularly in terms of false-positive findings by US we need to carry out further studies in less specialized hospitals.

\section{Conclusions}

US is a highly sensitive and specific imaging technique in diagnosing pediatric GI obstructions and their underlying causes. The combination of US and radiography may increase the diagnostic sensitivity and negative predictive value in such patients.

\section{Conflict of interest: none}

\section{References}

1. Hajivassiliou CA. Intestinal obstruction in neonatal/pediatric surgery. Semin Pediatr Surg 2003;12:241-253.

2. Juang D, Snyder CL. Neonatal bowel obstruction. Surg Clin North Am 2012;92:685-711.
3. Vasavada P. Ultrasound evaluation of acute abdominal emergencies in infants and children. Radiol Clin North Am 2004;42:445-456.

4. Fidler JL, Guimaraes L, Einstein DM. MR imaging of the small bowel. Radiographics 2009;29:1811-1825.

5. Hefny AF, Corr P, Abu-Zidan FM. The role of ultrasound in the management of intestinal obstruction. J Emerg Trauma Shock 2012;5:84-86.

6. van Randen A, Lameris W, van Es HW, et al. A comparison of the accuracy of ultrasound and computed tomography in common diagnoses causing acute abdominal pain. Eur Radiol 2011;21:1535-1545.

7. Puylaert JB. Ultrasonography of the acute abdomen: gastrointestinal conditions. Radiol Clin North Am 2003;41:12271242.

8. Musoke F, Kawooya MG, Kiguli-Malwadde E. Comparison between sonographic and plain radiography in the diagnosis of small bowel obstruction at Mulago Hospital, Uganda. East Afr Med J 2003;80:540-545.

9. Nylund K, Odegaard S, Hausken T, et al. Sonography of the small intestine. World J Gastroenterol 2009;15:1319-1330.

10. Kimmey MB, Martin RW, Haggitt RC, Wang KY, Franklin DW, Silverstein FE. Histologic correlates of gastrointestinal ultrasound images. Gastroenterology 1989;96:433-441.

11. Choe J, Wortman JR, Michaels A, Sarma A, Fulwadhva UP, Sodickson AD. Beyond appendicitis: ultrasound findings of acute bowel pathology. Emerg Radiol 2019;26:307317.

12. Esposito F, Di Serafino M, Mercogliano C, et al. The pediatric gastrointestinal tract: ultrasound findings in acute diseases. J Ultrasound 2019;22:409-442.

13. Garcia R, Arcement C, Hormaza L, et al. Use of the rectosigmoid index to diagnose Hirschsprung's disease. Clin Pediatr (Phila) 2007;46:59-63.

14. Gollub MJ, Yoon S, Smith LM, Moskowitz CS. Does the CT whirl sign really predict small bowel volvulus?: Experience in an oncologic population. J Comput Assist Tomogr 2006;30:25-32.

15. Maheshwari P, Abograra A, Shamam O. Sonographic evaluation of gastrointestinal obstruction in infants: a pictorial essay. J Pediatr Surg 2009;44:2037-2042.

16. Ogata M, Mateer JR, Condon RE. Prospective evaluation of abdominal sonography for the diagnosis of bowel obstruction. Ann Surg 1996;223:237-241.

17. Hucl T. Acute GI obstruction. Best Pract Res Clin Gastroenterol 2013;27:691-707.

18. Silva AC, Pimenta M, Guimaraes LS. Small bowel obstruction: what to look for. Radiographics 2009;29:423-439.

19. Jang TB, Schindler D, Kaji AH. Bedside ultrasonography for the detection of small bowel obstruction in the emergency department. Emerg Med J 2011;28:676-678.

20. Berlin SC, Goske MJ, Obuchowski N, et al. Small bowel obstruction in rats: diagnostic accuracy of sonography versus radiography. J Ultrasound Med 1998;17:497-504.

21. Richardson NG, Heriot AG, Kumar D, Joseph AE. Abdominal ultrasonography in the diagnosis of colonic cancer. $\mathrm{Br}$ J Surg 1998;85:530-533. 
22. Ko YT, Lim JH, Lee DH, Lee HW, Lim JW. Small bowel obstruction: sonographic evaluation. Radiology 1993;188:649-653.

23. Schmutz GR, Benko A, Fournier L, Peron JM, Morel E, Chiche L. Small bowel obstruction: role and contribution of sonography. European Radiology 1997;7:1054-1058.

24. Ikeda H, Matsuyama S, Suzuki N, Takahashi A, Kuroiwa M, Hatakeyama S. Small bowel obstruction in children: review of 10 years experience. Acta Paediatr Jpn 1993;35:504-507.

25. James V, Alsani FS, Fregonas C, Seguin J, Tessaro MO. Point-of-care ultrasound in pediatric small bowel obstruction: an ED case series. Am J Emerg Med 2016;34:2464. e1-2464.e2

26. Hwang JY. Emergency ultrasonography of the gastrointestinal tract of children. Ultrasonography 2017;36:204-221.

27. Trout AT, Towbin AJ, Fierke SR, Zhang B, Larson DB. Appendiceal diameter as a predictor of appendicitis in children: improved diagnosis with three diagnostic categories derived from a logistic predictive model. Eur Radiol 2015;25:2231-2238.

28. Pinto A, Pinto F, Faggian A, et al. Sources of error in emergency ultrasonography. Crit Ultrasound J 2013;5 Suppl $1: \mathrm{S} 1$

29. Gupta H, Dupuy DE. Advances in imaging of the acute abdomen. Surg Clin North Am 1997;77:1245-1263.

30. Chen SC, Lin FY, Hsieh YS, Chen WJ. Accuracy of ultrasonography in the diagnosis of peritonitis compared with the clinical impression of the surgeon. Arch Surg 2000;135:170-173.

31. Chavhan GB, Masrani S, Thakkar H, et al. Sonography in the diagnosis of pediatric gastrointestinal obstruction. $\mathrm{J}$ Clin Ultrasound 2004;32:190-199.

32. Kandasamy D, Sharma R, Gupta AK. Bowel Imaging in Children: Part 1. Indian J Pediatr 2019;86:805-816. 